\title{
Therapeutic Window of Photodynamic Treatment (PDT) in Conservative Periodontal Therapy -Analysis of Cell Migration Within A Three Dimensional Collagen Matrix-
}

\author{
Grimm WD ${ }^{1,2 *}$, Fritsch $\mathrm{T}^{2}$ and MA Vukovic ${ }^{2}$ \\ ${ }^{1}$ Periodontology, Department of Dentistry, Faculty of Health, Witten/Herdecke University, Witten, Germany \\ ${ }^{2}$ Stavropol State Medical University, Stavropol, Russia
}

*Corresponding author: Grimm WD Periodontology, Department of Dentistry, Faculty of Health, Witten/Herdecke University, Witten, Germany.

Received Date: May 07, 2021

Published Date: May 19, 2021

\section{Abstract}

Objectives: The aim of the present study was to investigate the effect of low-level laser irradiation as photodynamic therapy (PDT) on proliferation and differentiation of a human fibroblast cell line, determining the therapeutic window of toluidine blue 0 (TBO) using the 3-D collagen matrix assay with fibroblasts.

Material and methods: The collagen-based 3-D assay (MetaVi Labs, Bottrop, Germany) combines all the advantages of 3-D collagen matrix assays with the benefits of high-throughput, fully auto-mated (hands-off) tracking technology. Two sets of tests (MetaVì Labs 3-D collagen matrix assay) were performed with human dermal fibroblasts derived from PromoCell (Heidelberg, Germany). Cells do not need to be labelled with any dyes or tags that could interfere with cell motility or inter-act with substances being tested in the assay.

Results: The sensitization of fibroblasts with a physiological concentration of $5 \%$ (v:v) TBO and exposure to light from a $810 \mathrm{~nm}$ diode laser $(1 \mathrm{~min}, 0.3 \mathrm{~W}$ ) did not reduce cell viability and migratory activity measured by migration dynamics in comparison to the not sensitized fibroblasts.

Conclusion: The study provided evidence that PDT with a diode laser 810nm and the photosensitizer toluidine blue 0 does not influence locomotory behavior of human fibroblasts in vitro.

Keywords: Photodynamic therapy (pdt); Toluidine blue O (TBO); Human oral fibroblasts; 3-D collagen based migration assay; Cell activity

\section{Introduction}

It was previously found that photodynamic therapy (PDT) enhances anti-bacterial effect of conservative periodontal treatment (for overview see [1]). PDT can be defined as eradication of target cells by reactive oxygen species (ROS) produced by means of a photosensitizing compound and light of an appropriate wavelength. Although PDT is more widely known for its application to the treatment of neoplasms, there is also interest in antimicrobial PDT, as a large number of microorganisms (including oral species) have been reported to be killed in vitro by this approach [2]. PDT has been shown to be effective at killing the periodontopathic organisms responsible for infections in animal models and in clinical-controlled studies [3-5] Sgolastra et al.,
(2012). Furthermore, from these studies it appears that PDT using energy doses from $1.5 \mathrm{~J} / \mathrm{cm}^{2}$ to $5 \mathrm{~J} / \mathrm{cm}^{2}$ are capable of eliciting biostimulatory effects in vitro for adherent cells that may be useful in periodontal regeneration such as fibroblasts and neural crestrelated stem cells $[6,7]$.

Photosensitizers and photodynamic antimicrobial action

When an aromatic molecule absorbs light of a certain energy it may undergo an electronic transition to the singlet excited state (electron spins paired). The ability of a molecule to instigate redox reactions and/or to form singlet oxygen depends on the production of a sufficient population of triplet state molecules. This in turn 
depends on the decay rates of both the triplet and initially-formed singlet states. Photosensitizers are usually aromatic molecules which are efficient in the formation of long-lived triplet excited states. In terms of the energy absorbed by the aromatic-system, this again depends on the molecular structure involved:

-furocoumarin photosensitizers (psoralens) absorb relatively high energy ultraviolet (UV) light (approximately 300-350nm)

-macrocyclic, heteroaromatic molecules such as the phthalocyanines absorb lower energy, near-infrared light (approximately 700nm).

Some oral bacteria are susceptible to killing by laser light after their sensitization with toluidine blue 0 (TBO). The related phenothiazinium photosensitizer toluidine blue $\mathrm{O}$ (TBO) is considered here. Against Escherichia coli, TBO is known to be membrane active, since it causes increased permeability [8]. However the data may be to some extent contradictory. The clinical rationale for use of PDT in conservative periodontal treatment is that treatment is associated with anti-inflammatory effects [9] and with effects in wound healing [10]. This suggests the in vivo effects of PDT may be very complex, which to some extent should not be surprising. Factors affecting PDT in vivo actions would include degree of energy penetration through the tissue, the various absorption ability of cells in the various tissues, and complex chemical changes that maybe occurring in paracrine/autocrine manner.

Regardless of the complexity of in vivo situations, the fact that reproducible in vitro experiments demonstrate a biological effect provided support for us that there is some basis for PDT and it is not strictly an area of phenomenology. The development of a system for the lethal photosensitization of bacteria responsible for periodontal disease may be possible. The photodynamic therapy (PDT) using wavelengths of $810 \mathrm{~nm}$ in vivo would require a therapeutic window where bacteria could be killed without adjacent normal tissue damage.

\section{Proliferation and differentiation of human oral fibro- blasts evaluated in vitro}

The autonomous migration of specialized cells is an essential characteristic in both physiological and pathological functions in the adult human organism. Leukocytes, fibroblasts, and stem cells, but also tumor cells, are thus the subject of intense investigation in a broad range of research fields. A wide spectrum of methods have therefore been established to analyze chemokinetic and chemotactic cell migration, ranging from easy-to-handle two-dimensional surface migration assays to highly specialized three-dimensional and intravital analysis methods. To analyze cell migration several in vitro and in vivo cell migration assays have been developed in the past decades, including the Boyden chamber/transwell assay (Boyden, 1962), scratch assay/wound healing assay [11-13], threedimensional (3-D) collagen matrix migration assay [14] as well as intravital imaging/microscopy (for review see [15]).
In 3-D collagen matrix assays, the cells are embedded in a matrix of collagen, usually type I collagen because it is the predominant component of the extracellular matrix in the periodontium. Cell migration is continuously visualized using time-lapse video microscopy. This is typically done using a video camera mounted on a light microscope and coupled to a recording system such as a timelapse videorecorder. This method allows both maximum temporal resolution and long observation times. 3-D collagen assays are the best approximation of in vivo cell migration conditions. Therefore the aim of the present study was to investigate the effect of lowlevel laser irradiation as PDT on proliferation and differentiation of a human fibroblast cell line determining the therapeutic window of TBO using the 3-D collagen matrix assay with fibroblasts.

\section{Material and Methods}

For the assessment of viability and migratory activity of a human fibroblast cell line determining the therapeutic window of TBO, the 3-D collagen-based Migration Assay (MetaVi Labs, Bottrop, Ger-many) was used (Figure 1). The fibroblasts were continuously visualized after activating by TBO with a $810 \mathrm{~nm}$ diode laser using time-lapse video microscopy. The Percentile Endpoint Radius (indicating how far most of the observed fibroblasts moved within the observation time) and the Percent Migrated (showing the quantity of cells that are migrating each moment over time in a scene) were used comparing PDT-treated and untreated fibroblasts.

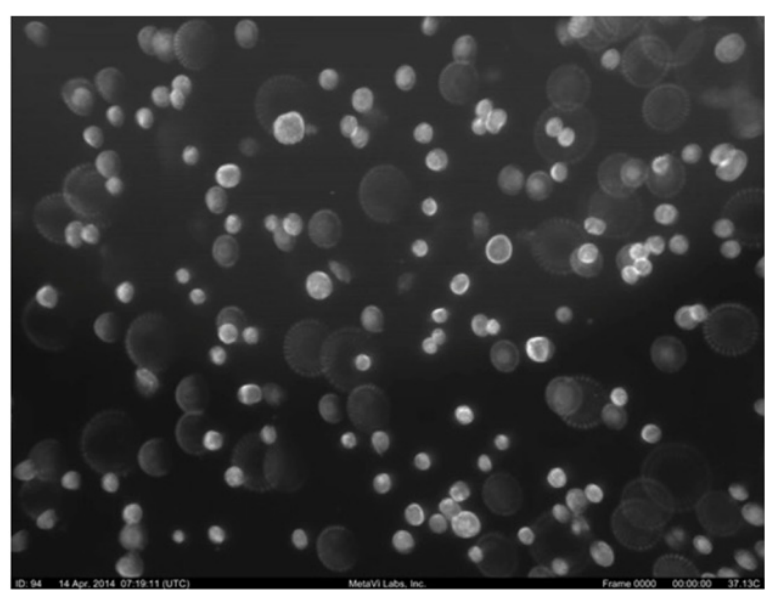

Figure 1: 3-D collagen-based Migration Assay assessing gingival fibroblasts.

\section{Cell lines}

Cultured fibroblast cells were irradiated using laser irradiation ( $810 \mathrm{~nm}$; $0.3 \mathrm{~mW}$ power output). On the second and third day after seeding the fibroblasts were exposed to laser irradiation. Two sets of tests (MetaVì Labs 3-D collagen Migration Assay) were performed with human dermal fibroblasts derived from PromoCell (Heidelberg, Germany). The cells were harvested at the indicated date and passage number below. After centrifugation 3 minutes at 220g), the cells were resuspended in $1 \mathrm{ml}$ PBS. Four aliquots were generated, each containing 60.000 cells. TBO (BluLase $^{\text {TM }}$ 
Sensitizer) or PBS were added to the same amount, resulting in TBO concentrations of $50 \%$ and $5 \%$ (v:v).

Two of each samples were treated with laser-light (BluLase ${ }^{\mathrm{TM}}$ $810 \mathrm{~nm}$ ) for 1 minute at 0.3 Watt (PDT, sensitization with toluidine blue $0\{\mathrm{TBO}\})$. After that resulting in a total incubation time of 5 minutes all samples were spun down and resuspended in 50 $\mu$ PBS. $100 \mu \mathrm{l}$ collagen solution was added to each sample, resulting in a collagen concentration of $1.67 \mathrm{mg} / \mathrm{ml}$. Locomotory behavior was analyzed for a 10 hours period:

-Set $1(50 \%$ TBO)
-Set 2 (5\% TBO)

\section{Results}

At a concentration of $50 \%$ TBO, the locomotor activity of the fibroblasts was significantly impaired, whereas 5\% TBO did not affect the part of locomoting cells (Figure 2, Table 1). PDT treatment had no influence on the locomotory behavior. Concomitantly, the speed of locomotion was significantly reduced in those cells treated with $50 \%$ TBO, but showed only a weak tendency to a reduced speed in those samples treated with 5\% TBO (Figure 3, Table 2). Again, PDT treatment did not influence the speed of locomotion in neither control samples nor TBO-treated samples.
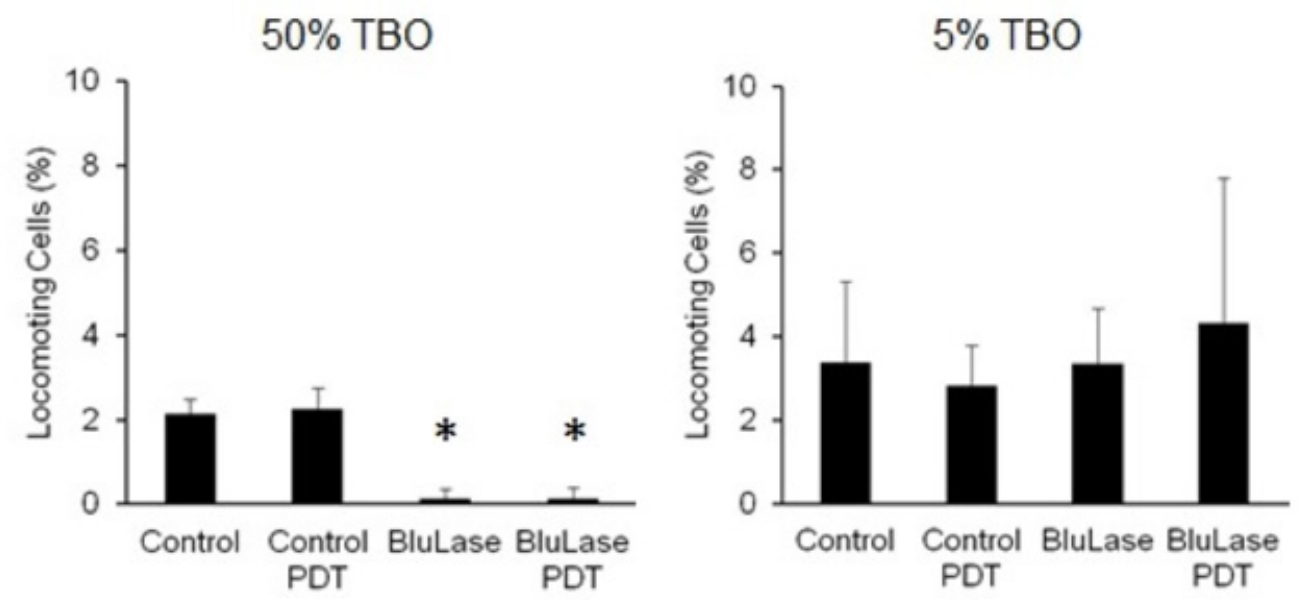

Figure 2: Percent migrated shows the quantity of cells that are migrating each moment over time. The source video is divided into 15 minute time intervals, and for each interval, all cells visible during that interval are categorized as either migrating (meaning they moved more than a threshold radius of $3 \mu \mathrm{m})$ or stationary.

Table 1: Percentage of migrating cells shows the quantity of cells that are migrating each moment over time.

\begin{tabular}{|c|c|c|c|c|}
\hline \multicolumn{5}{|c|}{ Set 1 (50\% ТВ0); \% of Migrating cells } \\
\hline $50 \%$ & Control & Control PDT & TBO & TBO PDT \\
\hline Mean & 2.12 & 2.27 & 0.14 & 0.14 \\
\hline SD & 0.38 & 0.49 & 0.24 & 0.25 \\
\hline T-Test & & & 0.0016 & 0.0017 \\
\hline \multicolumn{5}{|c|}{ Set 2 (5\% тВ0); $\%$ of Migrating cells } \\
\hline $5 \%$ & Control & Control PDT & Тво & TBO PDT \\
\hline Mean & 3.03 & 2.81 & 3.31 & 4.14 \\
\hline SD & 1.58 & 0.97 & 1.32 & 3.55 \\
\hline T-Test & & & 0.7916 & 0.5887 \\
\hline
\end{tabular}

According to the reduced speed of locomotion, the distance of movement that $95 \%$ of the observed cells reached, was significantly reduced at 50\% TBO (Figure 4, Table 3). The use of a percentile eliminates extreme outliers. As a combined analysis of locomotor activity and speed, the average distance that each cell of the observed populations travelled, was significantly reduced at 50\% TBO (Figure 5, Table 4), and showed a tendency to reduction at 5\% TBO without PDT treatment. For further analysis, the migratory activity was categorized into minimum distances reached by the migrating cells $(3,4,5,10,15$ and $20 \mu \mathrm{m})$. Interestingly, although $50 \%$ TBO led to a strong reduction of the migratory activity, there was still a small part of cells, which showed strong migratory activity comparable to untreated cells (Figure 6, Table 5). 5\% TBO only led to a slight reduction in those cells of moderate activity ( $4 \mu \mathrm{m}$ and $5 \mu \mathrm{m}$ distance reached). 

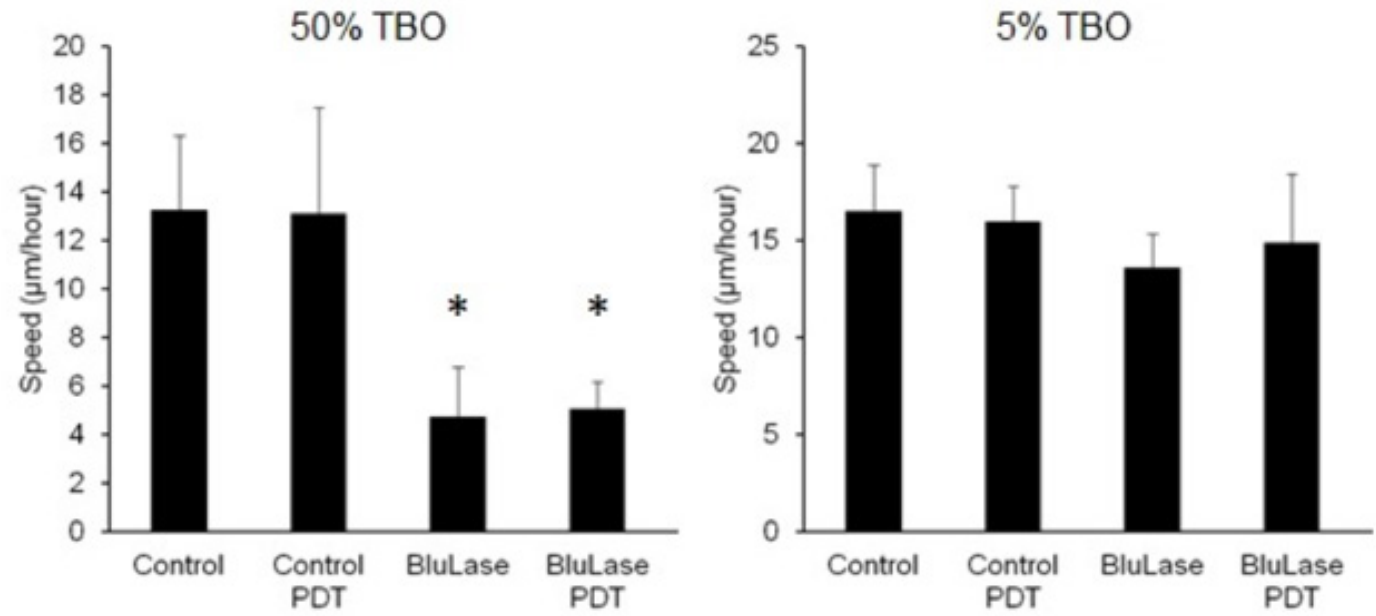

Figure 3: Total path length of all cells divided by the total time cells were visible.
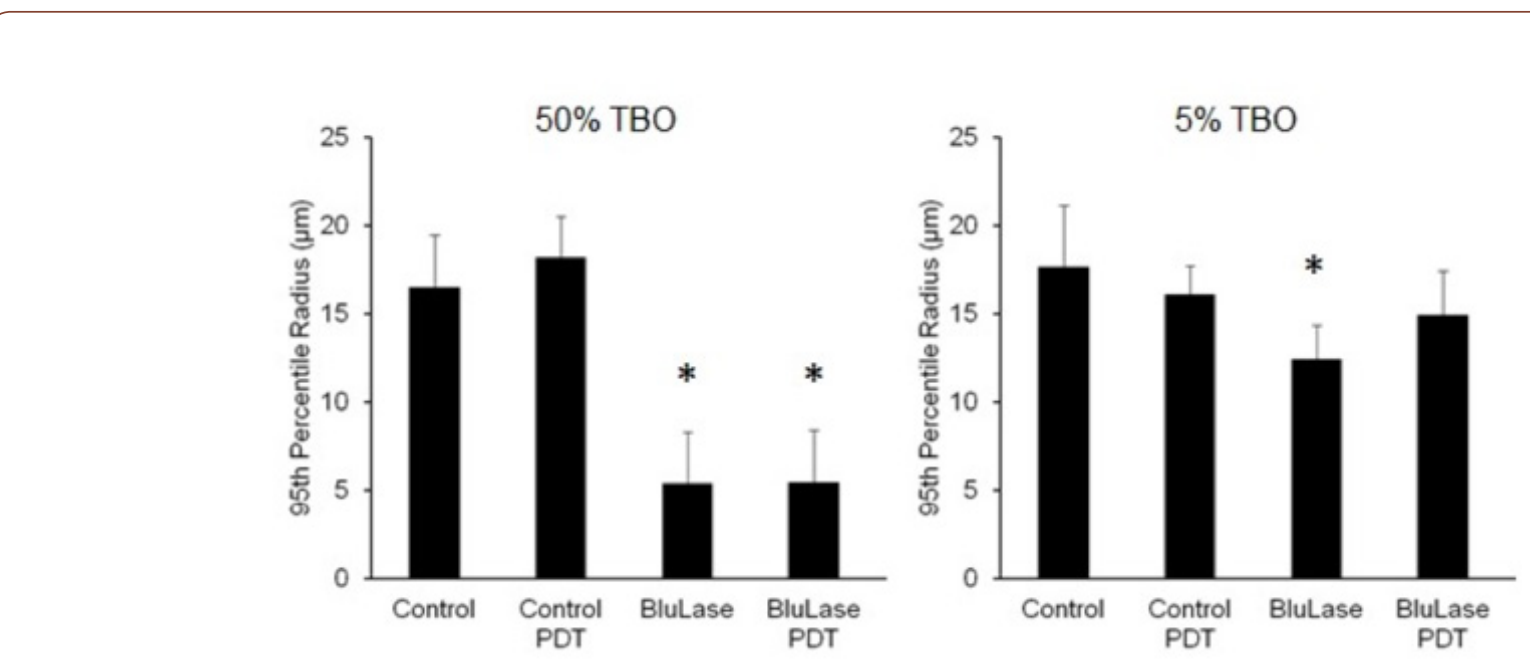

Figure 4: The percentile endpoint radius metric indicates how far most of the observed cells $(95 \%)$ moved within the observation time. Cell paths are sorted by their endpoint distance (the distance from their starting points to their starting points), and the radius that encompasses the shortest $95 \%$ of these cell path endpoint distances is calculated and plotted in the bar graph. The use of a percentile eliminates extreme outliers

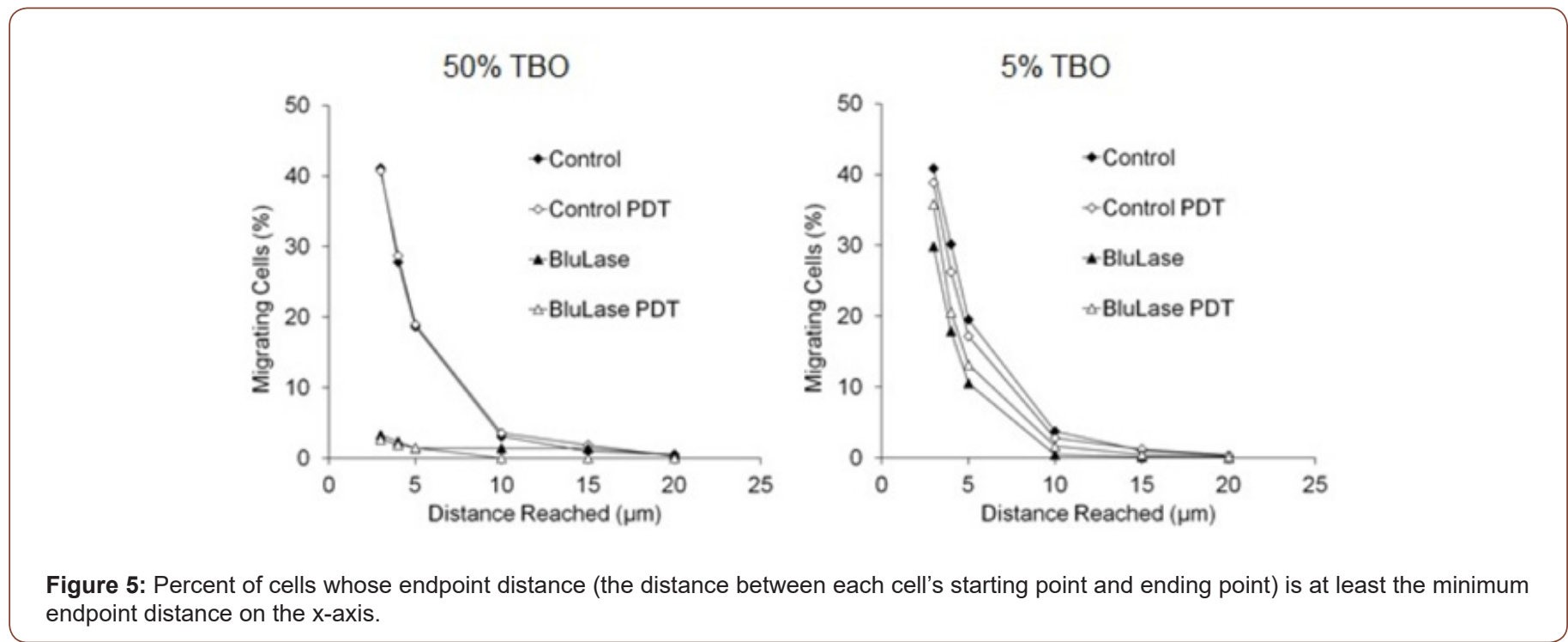


$50 \%$ TBO
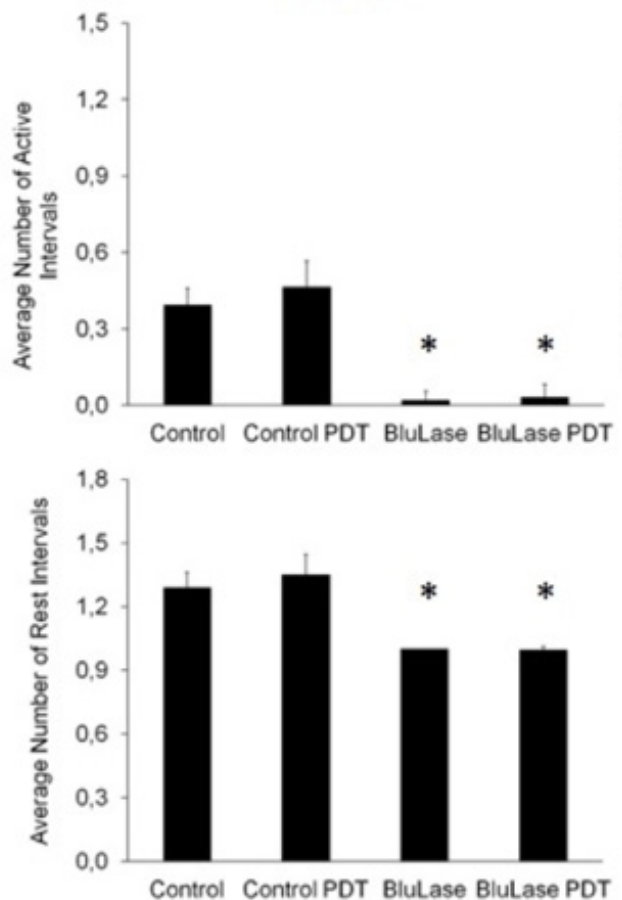

$5 \%$ TBO
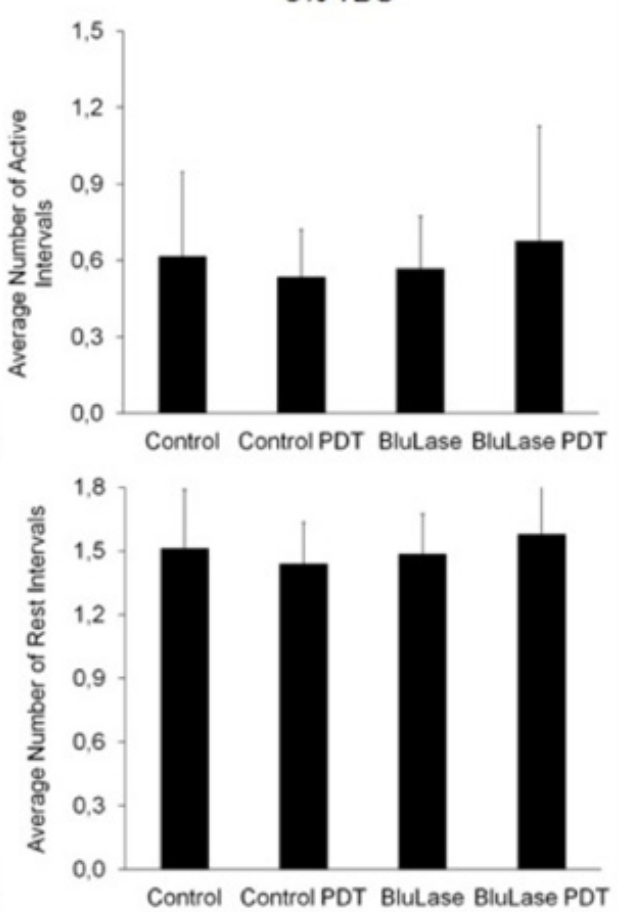

Figure 6: Average cell travel distance, with a 3.5 micron hysteresis threshold to eliminate noise.

Table 2: Total Path length of all cells divided by the total time.

Set 1 (50\% TB0); Total Path Length

\begin{tabular}{|c|c|c|c|c|}
\hline $50 \%$ & Control & Control PDT & ТВО & TBO PDT \\
\hline Mean & 13.25 & 13.09 & 4.77 & 5.09 \\
\hline SD & 3.06 & 4.39 & 2.01 & 1.13 \\
\hline T-test & & & 0.016 & 0.0123 \\
\hline \multicolumn{5}{|c|}{ Set 2 (5\% TBO); Total Path Length } \\
\hline $5 \%$ & Control & Control PDT & ТВО & TBO PDT \\
\hline Mean & 16.53 & 16 & 13.59 & 14.89 \\
\hline SD & 2.35 & 1.78 & 1.78 & 3.56 \\
\hline T-test & & & 0.0934 & 0.4698 \\
\hline
\end{tabular}

Table 3: Cell paths are sorted by their endpoint distance (the distance from their starting points to their starting points), and the radius that encompasses the shortest $95 \%$ of these cell path endpoint distances is calculated and plotted in the bar graph.

\begin{tabular}{|c|c|c|c|c|}
\hline \multicolumn{5}{|c|}{ Set 1 (50\% TB0); Percentile Endpoint Radius } \\
\hline $50 \%$ & Control & Control PDT & TBO & TBO PDT \\
\hline Mean & 16.51 & 18.2 & 5.43 & 5.49 \\
\hline SD & 2.96 & 2.32 & 2.91 & 2.95 \\
\hline T-test & & & 0.0099 & 0.0103 \\
\hline \multicolumn{5}{|c|}{ Set 2 (5\% TBO); Percentile Endpoint Radius } \\
\hline $5 \%$ & Control & Control PDT & TBO & TBO PDT \\
\hline Mean & 17.64 & 16.11 & 12.45 & 14.91 \\
\hline SD & 3.49 & 1.6 & 1.89 & 2.55 \\
\hline T-test & & & 0.0398 & 0.2527 \\
\hline
\end{tabular}


Table 4: Average cell travel distance.

\begin{tabular}{|c|c|c|c|c|}
\hline \multicolumn{5}{|c|}{ Set 1 (50\% TB0); Average cell travel distance } \\
\hline $50 \%$ & Control & Control PDT & ТВо & TBO PDT \\
\hline Mean & 2.27 & 2.17 & 0.4 & 0.12 \\
\hline SD & 0.26 & 0.39 & 0.69 & 0.19 \\
\hline T-test & & & 0.0115 & 0.0003 \\
\hline \multicolumn{5}{|c|}{ Set 2 (5\% TBO); Average cell travel distance } \\
\hline $5 \%$ & Control & Control PDT & ТВО & TBO PDT \\
\hline Mean & 2.36 & 2.04 & 1.42 & 1.84 \\
\hline SD & 0.81 & 0.39 & 0.6 & 1.12 \\
\hline T-test & & & 0.1107 & 0.4849 \\
\hline
\end{tabular}

Table 5: The distance between each cell's starting point and ending point.

\begin{tabular}{|c|c|c|c|c|}
\hline \multicolumn{5}{|c|}{ Set 1 (50\% TB0): Endpoint Distance } \\
\hline $50 \%$ & Control & Control PDT & тво & TBO PDT \\
\hline 3 & 41.13 & 40.76 & 3.29 & 2.71 \\
\hline 4 & 27.84 & 28.73 & 2.35 & 1.93 \\
\hline 5 & 18.64 & 18.97 & 1.41 & 1.45 \\
\hline 10 & 3.09 & 3.54 & 1.41 & 0 \\
\hline 15 & 0.99 & 1.87 & 1.41 & 0 \\
\hline 20 & 0.52 & 0.28 & 0.47 & 0 \\
\hline \multicolumn{5}{|c|}{ Set 2 (5\% TBO); Endpoint Distance } \\
\hline $5 \%$ & Control & Control PDT & тво & TBO PDT \\
\hline 3 & 40.85 & 38.86 & 29.83 & 35.77 \\
\hline 4 & 30.17 & 26.27 & 17.88 & 20.57 \\
\hline 5 & 19.52 & 17.18 & 10.55 & 13.16 \\
\hline 10 & 3.75 & 2.8 & 0.44 & 1.63 \\
\hline 15 & 1.02 & 1.19 & 0.16 & 0.46 \\
\hline 20 & 0.28 & 0.36 & 0 & 0.16 \\
\hline
\end{tabular}

The number of active intervals is significantly reduced by $50 \%$ TBO, and the number of rest intervals is concomitantly significantly reduced towards 1 (Figure 7, Table 6). This provides evidence that fewer cells are migrating in response to TBO, because each migrating cell must have at least one active interval, but those cells that are not migrating in the entire observation period have one rest interval. Therefore, the less the migratory activity in a population is, the closer the number of active intervals goes to 0 and the number of rest intervals goes to 1 . The average active interval length was reduced by $50 \%$ TBO, which however only reached statistical significance in combination with PDT due to the high standard deviation (Figure 8, Table 7). At 5\% TBO the average active interval length tended to be longer. In none of the test, the average length of rest intervals was significantly altered.

Table 6: Average number of active and rest intervals.

Set 1 (50\% TBO); Interval number

\begin{tabular}{|c|c|c|c|c|}
\hline \multicolumn{5}{|c|}{ Set 1 ( $50 \%$ TB0); Interval number } \\
\hline \multicolumn{5}{|c|}{ Active } \\
\hline $50 \%$ & Control & Control PDT & тво & TBO PDT \\
\hline Mean & 0.39 & 0.46 & 0.02 & 0.03 \\
\hline SD & 0.07 & 0.1 & 0.03 & 0.05 \\
\hline T-test & & & 0.001 & 0.0017 \\
\hline \multicolumn{5}{|c|}{ Set 2 ( $5 \%$ TBO); Interval number } \\
\hline \multicolumn{5}{|c|}{ Active } \\
\hline $5 \%$ & Control & Control PDT & ТВО & TBO PDT \\
\hline
\end{tabular}




\begin{tabular}{|c|c|c|c|c|}
\hline Mean & 0.62 & 0.54 & 0.57 & 0.68 \\
\hline SD & 0.33 & 0.18 & 0.2 & 0.45 \\
\hline T-test & & & 0.8154 & 0.8375 \\
\hline \multicolumn{5}{|c|}{ Set 1 ( $50 \%$ TBO); Interval number } \\
\hline \multicolumn{5}{|c|}{ Rest } \\
\hline $50 \%$ & Control & Control PDT & TBO & TBO PDT \\
\hline Mean & 1.29 & 1.35 & 1 & 1 \\
\hline SD & 0.07 & 0.1 & 0 & 0.02 \\
\hline T-test & & & 0.0022 & 0.0023 \\
\hline \multicolumn{5}{|c|}{ Set 2 ( $5 \%$ TBO); Interval number } \\
\hline \multicolumn{5}{|c|}{ Rest } \\
\hline $5 \%$ & Control & Control PDT & TBO & TBO PDT \\
\hline Mean & 1.51 & 1.44 & 1.49 & 1.58 \\
\hline SD & 0.28 & 0.19 & 0.19 & 0.41 \\
\hline T-test & & & 0.8862 & 0.7931 \\
\hline
\end{tabular}
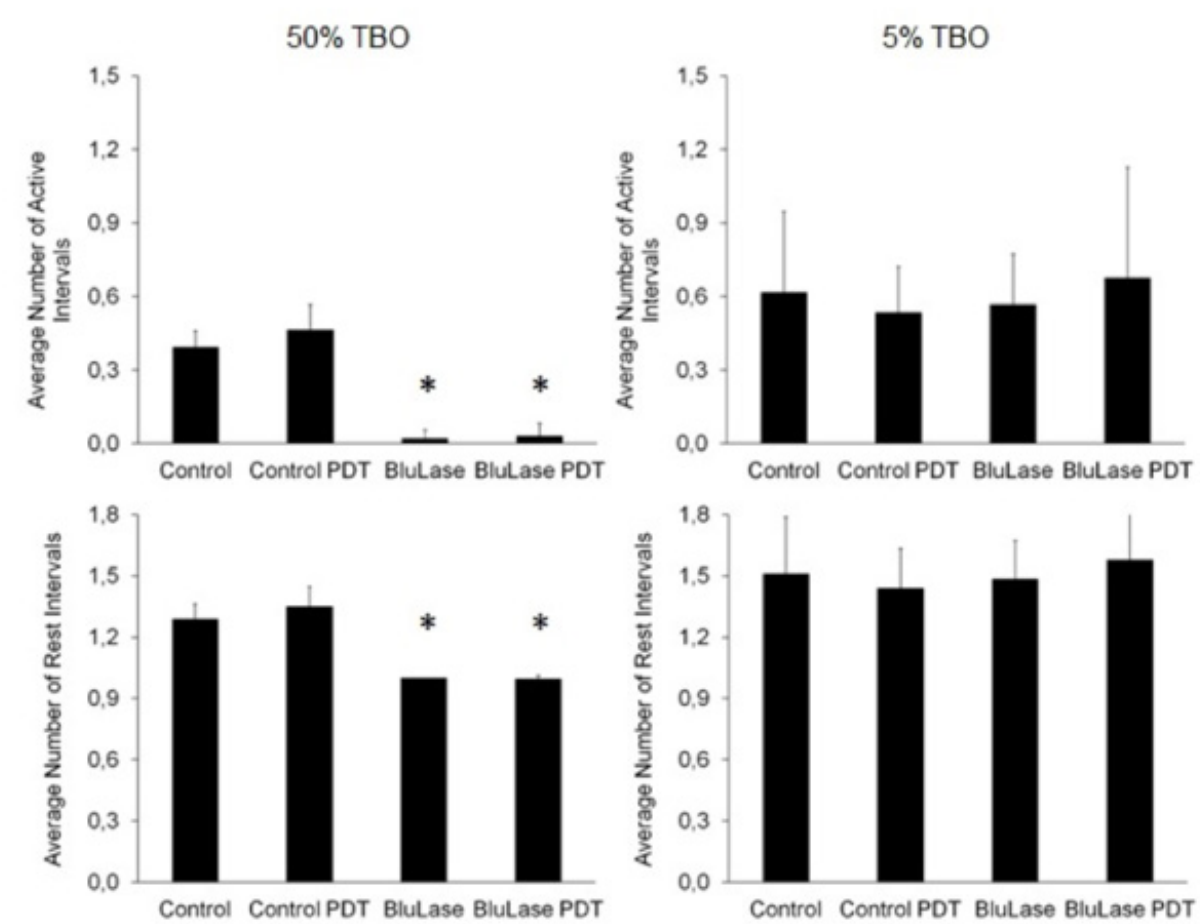

Figure 5: Average number of active and rest intervals.

Table 7: Average length of active and rest intervals.

\section{Set 1 (50\% TBO); Interval length}

\begin{tabular}{|c|c|c|c|c|}
\hline \multicolumn{5}{|c|}{ Active } \\
\hline $50 \%$ & Control & Control PDT & TBO & 0.63 \\
\hline Mean & 2.02 & 1.95 & 1.09 & 0.5 \\
\hline SD & 0.09 & 0.06 & 0.0917 & 0.0393 \\
\hline T-test & & & \\
\hline
\end{tabular}




\begin{tabular}{|c|c|c|c|c|}
\hline \multicolumn{5}{|c|}{ Active } \\
\hline $5 \%$ & Control & Control PDT & TBO & TBO PDT \\
\hline Mean & 2.06 & 1.98 & 2.3 & 2.18 \\
\hline SD & 0.17 & 0.07 & 0.26 & 0.4 \\
\hline T-test & & & 0.1626 & 0.5774 \\
\hline \multicolumn{5}{|c|}{ Set 1 ( $50 \%$ TBO); Interval length } \\
\hline \multicolumn{5}{|c|}{ Rest } \\
\hline $50 \%$ & Control & Control PDT & Тво & TBO PDT \\
\hline Mean & 28.86 & 29.48 & 23.93 & 25.49 \\
\hline SD & 6,26 & 3,52 & 9,48 & 8,15 \\
\hline T-test & & & 0,4941 & 0,5998 \\
\hline \multicolumn{5}{|c|}{ Set 2 (5\% ТВ0) Interval length } \\
\hline \multicolumn{5}{|c|}{ Rest } \\
\hline $5 \%$ & Control & Control PDT & TBO & TBO PDT \\
\hline Mean & 25,18 & 25,23 & 26,22 & 24,68 \\
\hline SD & 5,33 & 2,80 & 3,90 & 9,91 \\
\hline T-test & & & 0,7645 & 0,9314 \\
\hline
\end{tabular}

$50 \%$ TBO
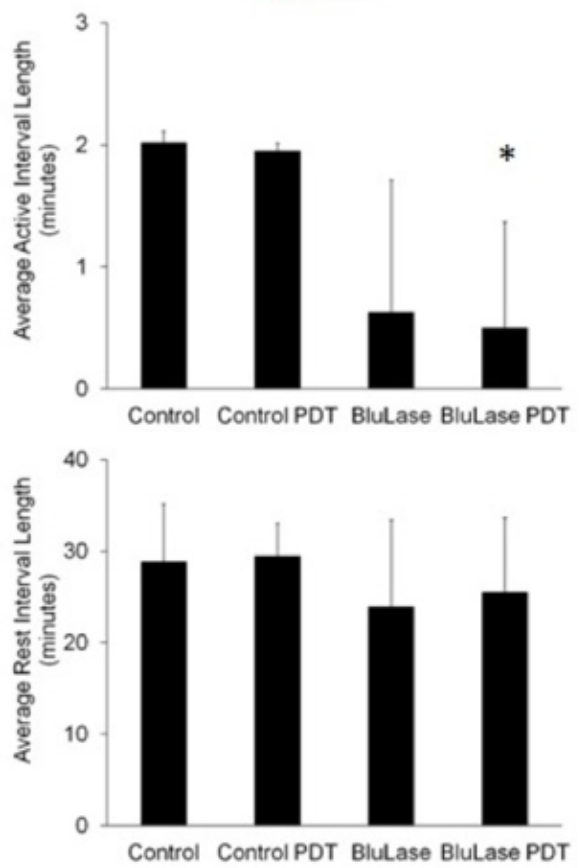

$5 \%$ TBO
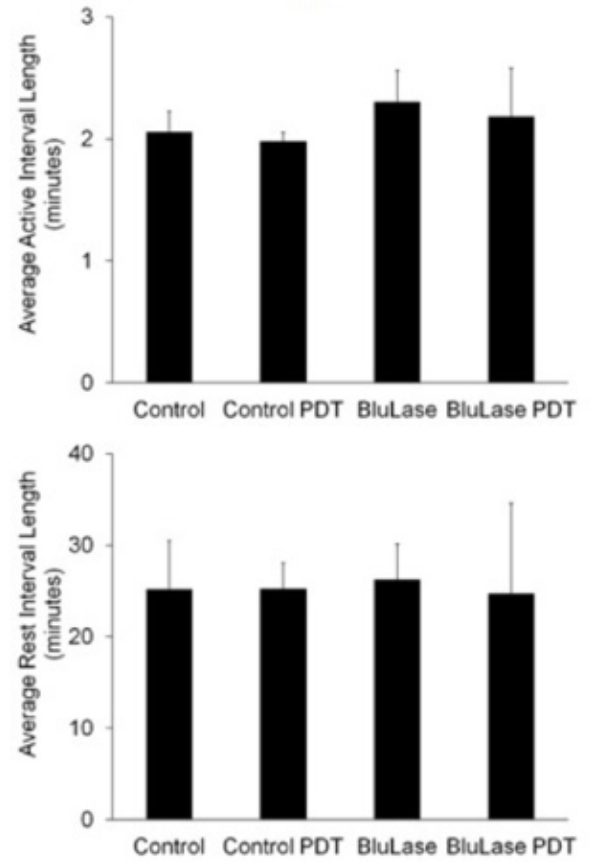

Figure 8: Average length of active and rest intervals.

\section{Discussion}

Major advances have been made in photodynamic therapy (PDT) in the past decade. Whilst it is not suggested that PDT will replace conservative periodontal treatment, improvements may be obtained using the photodynamic approach in the clinical treatment of local periodontal infection, both in terms of the likely speed of treatment and in the lowering of treatment cost. What is important in PDT, is the ability to excite the photosensitizer at its target site with minimal photo effect on the surrounding periodontal tissues. The main area which needs to be addressed is that of host toxicity, a therapeutic window where bacteria could be killed without adjacent normal periodontal tissue damage. 
Phenothiaziniums are blue dyes (600-660nm) such as methylene blue and TBO. Both meth-ylene blue and TBO are used in photodynamic therapy in periodontology and implantology $[16,17]$ at reasonably high concentrations (normally 1\% w/v) without causing human toxicity. In terms of PDT, it has been shown that the light dose required to kill bacteria treated with TBO is far lower than that causing toxicity in cultured human keratinocytes and fibroblasts. The size and shape of the phenothiaziniums, in line with other linear tricyclic heteroaromatics, make them ideal nucleic acid intercalators [18]. Thus, the site of action of methylene blue against E. coli is known to be DNA [8] whereas the closely related TBO is membrane-active [19].

The testing for cytotoxicity and cellular growth inhibition are the oldest and most commonly used assays to evaluate the efficacy of photosensitizers as a potential anti-bacterial method. In these in vitro assays, the amount of viable cells is usually tested on a panel cell lines over a wide range of concentrations. Although the investigation of cytotoxicity and cellular growth is often studied con-currently, there is a fundamental difference to be considered [20-22]. If a substance leads to a reduced number of viable cells in comparison to the untreated control after a certain incubation period, this can either be due to the death of some cells, but the unaffected growth of another subpopulation, or be due to a general deceleration of growth, but survival of all cells. Although the outcome of the net cell number might be the same in both variants, it is obvious that there is a basic difference in the method of action.

Therefore, several assays have been established to measure not only the viable cell number, but also to differentiate between the arrest of growth and cytotoxicity. However, such assays do not differentiate between the arrest of growth and cell death. There are of course many, not to say countless more in vitro methods for viability and cell death testing. In order to accommodate this broad spectrum of screening methods, there is no strict rule which set of methods has to be used for medical products, however there is a guideline of the International Organization for Standardization (ISO) for the evaluation of medical products (ISO 10993). In particular, ISO 10993-5 is applicable for the testing of in vitro cytotoxicity. This guideline does not stipulate which assays should be used, but sets a standard how test methods should be chosen.

The collagen-based 3-D assay (MetaVi Labs, Bottrop, Germany) combines all the advantages of 3-D collagen matrix assays with the benefits of high-throughput, fully automated (hands-off) tracking technology. Cells do not need to be labelled with any dyes or tags that could interfere with cell motility or interact with substances being tested in the assay. Whole cell populations, thousands of cells, can be tracked simultaneously in real- time. MetaVì Labs' analysis software can analyze migration and morphology data by population, by sub-population, or by closer examination of individual cells. The collagen-based 3-D assay is the only validated, consistent cell migration assay available for high volume preclinical screening applications. With this collagen-based 3-D assay, the overall anticellular effect of substance can be evaluated on fibroblast cell lines with metrics that can be used for standardized comparison.

\section{Conclusion}

High concentrations (50\%) of TBO affect the locomotory behavior of human fibroblasts, whereas concentrations that are supposed to occur in physiological conditions ( $5 \%$ and below) have only weak influence on the locomotory behavior. PDT treatment does not influence locomotory behavior of human fibroblasts. We conclude that PDT promotes proliferation and maturation of human fibroblasts in vitro.

\section{Acknowledgment}

The authors thank Prof. F. Entschladen for providing the collagen-based 3-D assay (MetaVi Labs, Bottrop, Germany).

\section{Conflict of Interest}

The authors declare no conflict of interest.

\section{References}

1. Herrera D (2011) Photodynamic therapy for chronic periodontitis. Evidence Based Dentistry 12: 78-79.

2. Konopka K, Goslinski T (2007) Photodynamic therapy in dentistry. J Dent Res 86: 694-707.

3. Chondros P, Nikolidakis D, Christodoulides N (2009) Photodynamic therapy as adjunct to non-surgical periodontal treatment in patients on periodontal maintenance: a randomized controlled clinical trial. Lasers in Med Sci 24: 681-688.

4. Christodoulides N, Nikolidakis D, Chondros P (2008) Photodynamic therapy as an adjunct to non-surgical periodontal treatment: a randomized, controlled clinical trial. J Periodontol 79: 1638-1644.

5. Takasaki AA, Aoki A, Mizutani K (2009) Application of antimicrobial photodynamic therapy in periodontal and peri-implant diseases. Periodontol 2000 51: 109-140.

6. Grimm WD, Giesenhagen B, Hakki S (2015) Translational Research and Therapeutic Applications of Neural Crest-Derived Stem Cells in Regenerative Periodontology. Current Oral Health Report.

7. Arany PR, Cho A, Hunt TD, Sidhu G (2014) Photoactivation of endogenous latent transforming growth factor- $\beta 1$ directs dental stem cell differentiation for regeneration. Sci Transl Med 28: 6(238): 238ra69.

8. Menezes S, Capella MA, Caldas LR (1990) Photodynamic action of methylene blue: repair and mutation in Escherichia coli. J Photochem Photobiol B 5: 505-517

9. Eick S, Markauskaite G, Nietzsche S (2013) Effect of photoactivated disinfection with a light-emitting diode on bacterial species and biofilms associated with periodontitis and peri-implantitis. Photodiagnosis Photodyn Ther 10: 156-167.

10. Oyama J, Ramos-Milaré A, Lera-Nonose D, Nesi-Reis V, Demarchi I, et al. (2020) Photodynamic therapy in wound healing in vivo: a systematic review. Photodiagnosis Photodyn Ther 30: 101682

11. Haudenschild CC, Schwartz SM (1979) Endothelial regeneration. II. Restitution of endothelial continuity. Lab Invest 41: 407-418.

12. Todaro GJ, Lazar GK, Green H (1965) The initiation of cell division in a contact-inhibited mammalian cell line. J Cell Physiol 66: 325-333.

13. Liang CC, Park AY, Guan JL (2007) In vitro scratch assay: a convenient and inexpensive meth-od for analysis of cell migration in vitro. Nat Protoc 2:329-333. 
14. Friedl P, Noble PB, Zänker KS (1993) Lymphocyte locomotion in threedimensional collagen gels. Comparison of three quantitative methods for analysing cell trajectories. J Immunol Methods 165: 157-165.

15. Pittet MJ, Weissleder R (2011) Intravital imaging. Cell 147: 983-991.

16. Mielczarek-Badora E, Szulc M (2013) Photodynamic therapy and its role in periodontitis treat-ment. Postepy Hig Med Dosw (Online) 13: 10581065 .

17. Vohra F, Al-Rifaiy MQ Lillywhite G (2014) Efficacy of mechanical debridement with adjunct antimicrobial photodynamic therapy for the management of peri-implant diseases: a systematic review. Photochem Photobiol Sci 13: 1160-1168.

18. Soukos NS, Wilson M, Burns T (1996) Photodynamic effects of toluidine blue on human oral keratinocytes and fibroblasts and Streptococcus sanguis evaluated in vitro. Lasers Surg Med 18: 253-259.
19. Wakayama Y, Takagi M, Yano K (1980) Photosensitized inactivation of E. coli cells in toluidine blue-light system. Photochem Photobiol 32: 601615.

20. Park D, Kim M, Choi JW, Baek JH, Lee SH, et al. (2020) Antimicrobial photodynamic therapy ef-ficacy against specific pathogenic periodontitis bacterial species. Photodiagnosis Photodyn Ther 30: 101688.

21. Pittet MJ, Weissleder R (2011) Intravital imaging. Cell 147: 983-991.

22. Sgolastra F, Severino M, Gatto R (2013) Effectiveness of diode laser as adjunctive therapy to scaling root planning in the treatment of chronic periodontitis: a meta-analysis. Lasers Med Sci 28: 1393-1402. 